\title{
Estimating the adaptive potential of critical thermal limits: methodological problems and evolutionary implications
}

\author{
Enrico L. Rezende ${ }^{1}$, Miguel Tejedo ${ }^{2}$ and Mauro Santos ${ }^{\star, 1}$ \\ ${ }^{1}$ Departament de Genètica i de Microbiologia, Grup de Biologia Evolutiva (GBE), Universitat Autònoma de Barcelona, \\ 08193 Bellaterra (Barcelona), Spain; and ²Department of Evolutionary Ecology, Estación Biológica de Doñana-CSIC, \\ Avda. Américo Vespucio s/n, E-41092 Sevilla, Spain
}

\begin{abstract}
Summary
1. Current studies indicate that estimates of thermal tolerance limits in ectotherms depend on the experimental protocol used, with slower and presumably more ecologically relevant rates of warming negatively affecting the upper thermal limits $\left(\mathrm{CT}_{\max }\right)$. Recent empirical evidence also gives credence to earlier speculations suggesting that estimates of heritability could drop with slower heating rates.

2. Using published data from the fruit fly Drosophila melanogaster, we show that empirical patterns can be explained if flies' physical condition decreases with experimental time in thermal tolerance assays. This problem could even overshadow potential benefits of thermal acclimation, also suggesting that a drop in $\mathrm{CT}_{\max }$ with slower heating rates does not necessarily rule out beneficial acclimatory responses.

3. Numerical results from a simple illustrative model show that no clear conclusions can be obtained on how the phenotypic variance in $\mathrm{CT}_{\max }$ will be affected with different rates of thermal change. Conversely, the genetic variance and estimated heritabilities are expected to drop with slower heating rates, hence ramping rates in experiments aiming to study the evolutionary potential of thermal tolerance to respond to global warming should be as fast as possible (within the range in which measurement accuracy and physical condition are not affected).

4. Measurements under ecologically realistic warming rates should also consider the impact of other physiological and behavioural strategies that might partly compensate the negative effects of slow heating rates. However, there are situations in which slow heating rates closely mimic natural conditions, as those encountered by some aquatic ectotherms. These heating rates may be an issue of major concern in these species, given its negative impact on $\mathrm{CT}_{\max }$ and its adaptive potential.
\end{abstract}

Key-words: ectotherms, energy expenditure, evolvability, heritability, phenotypic plasticity, thermal ramping, thermal tolerance

The reason why plants and animals (including men) are so sensitive to temperature is that they are chemical machines .... Many of them do burn even at ordinary temperatures, but so slowly that we don't notice any great change even within a lifetime. (Haldane 1940, pp. 69-70)

\section{Introduction}

Body temperature affects metabolic rates and has a major impact on an ectotherm's short-term performance (i.e. any measure of an organism's capacity to function), and eventually on its fitness (Huey \& Kingsolver 1989; Hochachka \& Somero 2002; Martin \& Huey 2008; Angilletta 2009). Thermal performance is described by a curve (Huey \& Stevenson 1979), which rises gradually with temperature

*Correspondence author. E-mail: mauro.santos@uab.es 
from a critical thermal minimum $\left(\mathrm{CT}_{\min }\right)$ to an optimum temperature $\left(\mathrm{T}_{\mathrm{opt}}\right)$, and then drops rapidly to the critical thermal maximum $\left(\mathrm{CT}_{\max }\right)$. In the short term the thermal extremes that an organism can tolerate will typically depend on phenotypic plasticity, but long-term evolutionary shifts in thermal limits obviously depend on the presence of additive genetic variance.

Current interest in understanding species' thermal physiological limits has been fostered to some extent by pressing concerns with human-induced climate change. Reliable projections of its biological impacts should be based not only on average predictions, but also on the magnitude of the climatic fluctuations in time. One worrying conclusion that emerges from recent work is that tropical species are currently closer to their upper thermal limits (Deutsch et al. 2008; Tewksbury, Huey \& Deutsch 2008; Huey et al. 2009), and that temperate species might encounter extreme conditions in relatively short periods of time (Hoffmann 2010). Recent projections predict that warming will drive about $40 \%$ of lizard populations to extinction before the end of the century, although mainly because a reduction in time available for fitness-enhancing activities such as growth, foraging, etc. (Sinervo et al. 2010). In any case, obtaining realistic estimates of upper thermal tolerance limits and their evolvability/heritability is an important endeavour to provide a reliable assessment of species' extinction risk to global warming. Current work indicates that $\mathrm{CT}_{\max }$, as well as its plasticity and evolutionary potential, can be overestimated in those situations when heating rates in thermal tolerance assays are higher than natural ones (Terblanche et al. 2007; Chown et al. 2009). This adds more pessimism to an already alarming situation.

Thermal tolerance is usually quantified with two different experimental methods, labelled 'static' and 'dynamic'. In the static method, organisms are placed acutely at a stressful temperature and time to physical incapacitation ('knockdown time') is recorded. In the dynamic method ('ramping', sensu Mitchell \& Hoffmann 2010), temperature is increased or decreased gradually until individuals reach their knockdown temperature. The classical dynamic method (Cowles \& Bogert 1944) is appealing because it provides a direct estimate of the target variables $\left(\mathrm{CT}_{\max }\right.$ or $\left.\mathrm{CT}_{\text {min }}\right)$, and rates of thermal change can be adjusted to render ecologically realistic values (Terblanche et al. 2007). The problem is that both the average and variance of the estimated thermal limits can change with different heating or cooling rates (Mora \& Maya 2006; Terblanche et al. 2007; Chown et al. 2009). For example, $\mathrm{CT}_{\max }$ has been shown to rise with faster heating rates in the fish Acantemblemaria hancocki (Mora \& Maya 2006), in the tsetse fly Glossina pallipides (Terblanche et al. 2007), in the fruit fly Drosophila melanogaster (Chown et al. 2009), and in the Argentine ant Linepithema humile (Chown et al. 2009); a puzzling result because slower heating rates should allow individuals to acclimatize to new temperatures and because slow

heating pre-exposes individuals to non-lethal high temperatures ('hardening'), which increases heat shock resistance (see e.g. Hoffmann, Sørensen \& Loeschcke 2003). Also disturbing and presumably more realistic, heating rates (Mitchell \& Hoffmann 2010), which somewhat substantiates previous speculations (Chown et al. 2009).

Here, we argue that there are some fundamental problems in comparing the evolutionary potential of thermal limits assessed in dynamic methods with different rates of thermal change. We focus primarily on D. melanogaster, given our familiarity with this model and the abundant information on the physiology and evolution of tolerance limits in this species (reviewed in Hoffmann, Sørensen \& Loeschcke 2003), which allows (i) calculating the impact of different ramping protocols on thermal limits employing parameters estimated empirically and (ii) contrasting our theoretical predictions against experimental outcomes described in the literature. However, we emphasize that the rationale employed here and its repercussions should be general to ectothermic animals, even though the factors potentially involved will depend on the model and the experimental setting employed (see below). We show why decreasing heating rates are expected to negatively affect $\mathrm{CT}_{\max }$ and estimated heritabilities, and also obscure potentially beneficial plastic responses. Although we illustrate our arguments with quantitative analyses and numerical results for heuristic purposes, our main concern here is to be biologically realistic rather than quantitatively precise.

\section{Effects of 'ramping' on energy expenditure}

When upper thermal limits are assessed by gradually increasing temperature from an initial temperature $\mathrm{T}_{0}$ to the critical thermal maximum $\mathrm{CT}_{\max }$ at a rate $\mathrm{DT}\left({ }^{\circ} \mathrm{C} \min { }^{1}\right)$, the protocol imposes $\mathrm{CT}_{\max }$ to be co-linear with experimental time

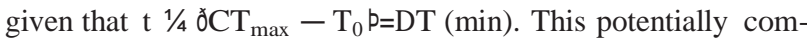
plicates or even invalidates the analysis of causal associations because 'not everything else is equal', as required by principles of experimental design. Among other things, the accumulated metabolic costs of individuals under different ramping regimes are not equal; hence their physical conditions and capability to cope with increasing temperatures should eventually decrease with time.

To demonstrate this problem in D. melanogaster, we first estimate the total energy expenditure associated with different regimes to measure $\mathrm{CT}_{\max }$. Temperature-dependence metabolic rate (MR) for a given taxon can be quantified in terms of the traditional $\mathrm{Q}_{10}$ factor, which is defined by the equation

$\mathrm{Q}_{10}{ }^{1 / 4}{\frac{\mathrm{MR} \delta \mathrm{T}_{1} \mathrm{p}}{\mathrm{MR} \delta \mathrm{T}_{0} \mathrm{p}}}^{10=\delta \mathrm{T}_{1}-\mathrm{T}_{0} \mathrm{p}}$

eqn1

where temperatures $\left(\mathrm{T}_{1}>\mathrm{T}_{0}\right)$ are expressed in ${ }^{\circ} \mathrm{C}$ and $\mathrm{Q}_{10}$ is considered 'constant' (but see below). Metabolic rates throughout the heat resistance assay with heating rate DT can be estimated from eqn 1 as

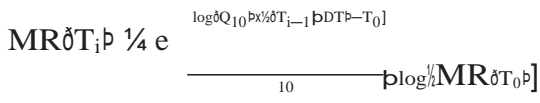

eqn2

is the recent finding that heritability drops to zero with slow 
where log is the natural logarithm, $\left(\mathrm{T}_{\mathrm{i}) 1}+\mathrm{DT}\right)>\mathrm{T}_{0}$ is the temperature at the ith minute from the start of the 
experiment, and $\operatorname{MR}\left(\mathrm{T}_{0}\right)$ is the metabolic rate at the initial temperature $\mathrm{T}_{0}$. The total energy expenditure of an individual knocked down at the nth minute can be simply obtained from the vector of $\mathrm{MR}\left(\mathrm{T}_{\mathrm{i}}\right)$ values resulting from eqn 2 as

EC $1 / 4_{\mathrm{i}^{1} / 41}^{X} \operatorname{MR}_{\mathrm{T}} \mathrm{T}_{\mathrm{i}} \mathrm{p}$ :

eqn3

It is immediately clear that the metabolic cost to reach $\mathrm{CT}_{\max }$ will be increasingly higher with decreasing DT, everything else being equal. Therefore, a simple consideration on the impact of metabolic costs on a fly's resistance to increasing temperatures (see below) predicts $\mathrm{CT}_{\max }$ to be positively correlated with DT (at least within certain limits), which is precisely what has been empirically observed in several experiments (Mora \& Maya 2006; Terblanche et al. 2007; Chown et al. 2009; Peck et al. 2009).

We have quantified the total amount of energy spent during the experimental trials performed by Chown et al. (2009) and Mitchell \& Hoffmann (2010) to illustrate how much of a problem this can entail to an 'average' fruit fly weighting $1 \mathrm{mg}$ with a metabolic rate of $4\left[2 \mathrm{~mL} \mathrm{O}_{2} \mathrm{~g}^{{ }^{1}} \mathrm{~h}^{{ }^{1}}\right.$ at $18{ }^{\circ} \mathrm{C}$ (Berrigan \& Partridge 1997). We assumed that metabolic rates increase with temperature with $\mathrm{Q}_{10}$ of 1579 and 2 $₫ 5$, which correspond to the extreme values reported in Berrigan \& Partridge (1997). All values employed in calculations and resulting estimates of energy expenditure are listed in Table 1. For example, in the experiments with D. melanogaster females described in Chown et al. (2009), the flies were individually placed in sealed empty vials immersed in a water-bath at an initial temperature $\mathrm{T}_{0}=20^{\circ} \mathrm{C}$. After an equilibration period of $6 \mathrm{~min}$ (ignored in subsequent calculations for simplicity), temperatures increased at three different heating rates of $0 \llbracket 1,0 \llbracket 25$ or $0\left[5^{\circ} \mathrm{C} \mathrm{min}{ }^{1}\right.$. As mean $\mathrm{CT}_{\max }$ at the slowest heating rate of $\left.\mathrm{DT}=0 \llbracket 1{ }^{\circ} \mathrm{C} \mathrm{min}\right)^{1}$ was approximately $38 \llbracket 7^{\circ} \mathrm{C}$ (see fig. 1a in Chown et al. 2009), the assay lasted $187 \mathrm{~min}$ before the average fly was knocked down. Conversely, the trial with a heating rate of DT $=0\left\lfloor 5{ }^{\circ} \mathrm{C} \mathrm{min}{ }^{1}\right.$ and average estimated $\mathrm{CT}_{\max }=39: 9^{\circ} \mathrm{C}$ lasted $39 \mathrm{~min}$. As a result, flies in the slow heating rate measurement consumed about four times more energy than their counterparts in the fast heating rate assay (Fig. 1). The same effect is observed in measurements of $\mathrm{CT}_{\text {min }}$ estimated with different cooling rates, even though in this case the net amount of energy spent during trials is substantially lower than in assays of $\mathrm{CT}_{\max }$ (Fig. 1).

Analyses employing values listed in Mitchell \& Hoffmann (2010) for D. melanogaster flies derived from two Australian populations (Gordonvale, Queensland; and Melbourne, Victoria) provide similar results in spite of some methodological differences. In one experiment, $\mathrm{CT}_{\max }$ was estimated as the knockdown time in flies subjected to a ramping trial with $\mathrm{T}_{0}=28^{\circ} \mathrm{C}$ and initial equilibration period of $15 \mathrm{~min}$ (also obviated in subsequent calculations). Temperature was then increased at a rate DT $\left.=0 \because 06{ }^{\circ} \mathrm{C} \mathrm{min}\right)^{1}$. In the other experiment, time to knockdown was estimated at a constant (static)

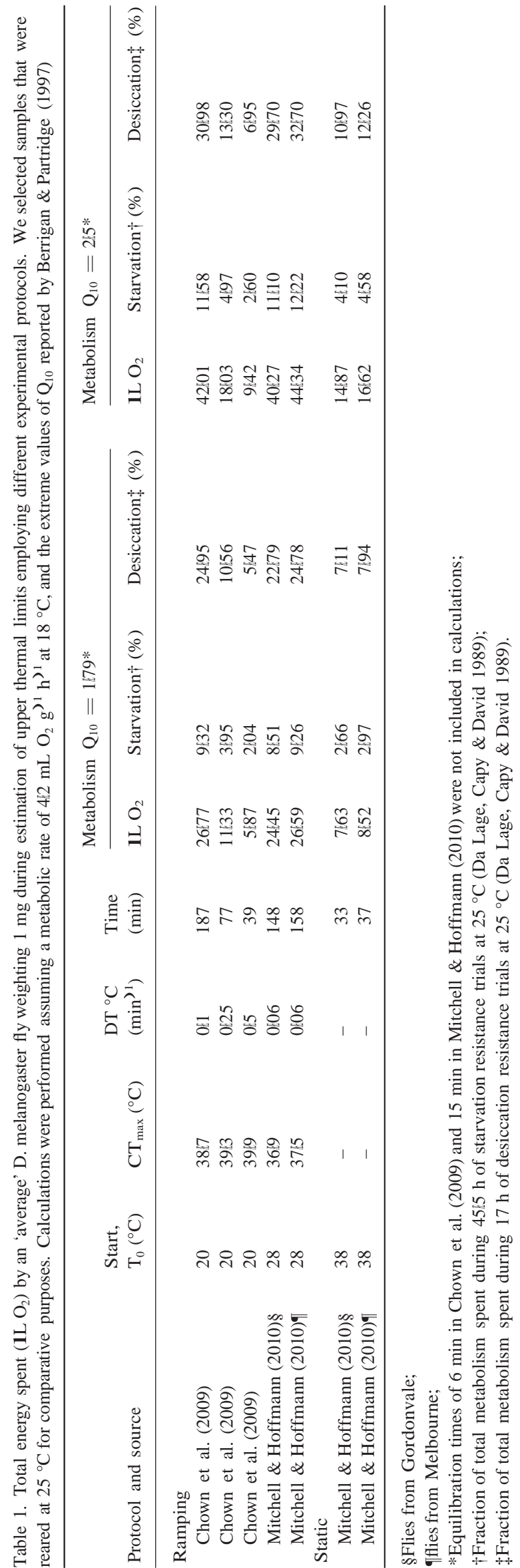

(C) 2010 The Authors. Functional Ecology (C) 2010 British Ecological Society, Functional Ecology, 25, 111-121 

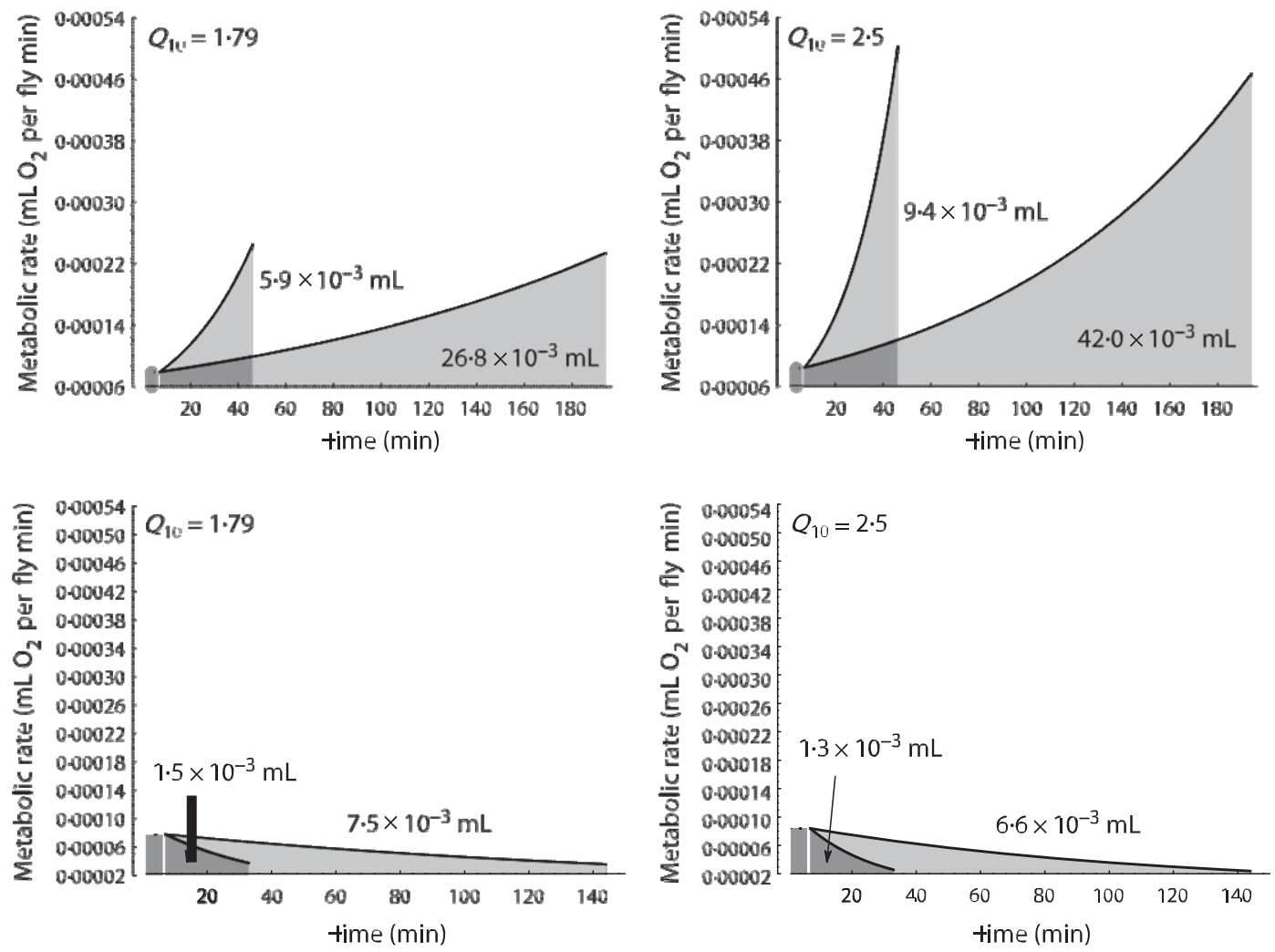

Fig. 1. Estimated metabolic rates from eqn 2 (black lines), and total energy expenditure from eqn 3 (shaded areas), of an average D. melanogaster fly weighting $1 \mathrm{mg}$ in the ramping experiments performed by Chown et al. (2009). Calculations assumed a metabolic rate of $4{ }^{\sharp 2} \mathrm{~mL} \mathrm{O}_{2} \mathrm{~g}^{{ }^{1}} \mathrm{~h}^{{ }^{1}}$ at $18^{\circ} \mathrm{C}$ (Berrigan \& Partridge 1997). Upper panels: $\mathrm{CT}_{\max }$ assays with $\mathrm{DT}=0 \llbracket 1^{\circ} \mathrm{C}$ min ${ }^{1}$ (lasting for 187 min before the fly was knocked down), and DT $=0: 5{ }^{\circ} \mathrm{C} \mathrm{min}^{1}$ (lasting for $39 \mathrm{~min}$ ), using the two extremes $\mathrm{Q}_{10}$ values reported by Berrigan \& Partridge (1997). Lower panels: $\mathrm{CT}_{\min }$ assays with cooling rates $\mathrm{DT}=0: 1{ }^{\circ} \mathrm{C} \mathrm{min}^{1}$, and $\mathrm{DT}=0: 5{ }^{\circ} \mathrm{C}$ min ${ }^{1}$. Shaded areas below the dotted points at the start of the experiments are the estimated energy expenditures during the equilibration period of 6 min (not taken into account for subsequent calculations in Table 1).

stressful temperature of $38^{\circ} \mathrm{C}$. Calculations suggest an approximate threefold difference in total energy expenditure, being it higher in the ramping than in the static assay (Fig. 2).

However, the preceding analyses could be criticized on theoretical grounds because they assume that $\mathrm{Q}_{10}$ in eqn 2 is constant, and a thermodynamically correct expression for $\mathrm{Q}_{10}$ shows it is a function of and changes with temperature (Schmidt-Nielsen 1997; Gillooly et al. 2001). We have also performed computations with different, more 'realistic', $\mathrm{Q}_{10}$ functions and results are given in Appendix S1 (Supporting Information). If anything, these analyses even strengthen the conclusions to be reached in the manuscript assuming $\mathrm{Q}_{10}$ constant in eqn 2, as we will do henceforth.

Note also that calculation in Figs 1 and 2 assume that metabolic rates are not lowered when flies are under food and water deprivation in sealed empty vials, which seems to be the case in D. melanogaster (Djawdan, Rose \& Bradley 1997). They also ignore the increased consumption of energy for protein production following a heat shock (Clark 2004). Furthermore, the maximum estimated $\mathrm{O}_{2}$ consumption in Table 1 represents a negligible fraction $(<1 \%)$ of vial's volume (5 mL in Mitchell \& Hoffmann 2010; $10 \mathrm{~mL}$ in Chown et al. 2009) and, hence, hypoxia was probably not a problem. It remains to be seen, however, how much of an impact the previous estimates of energy expenditure represent to total energy and water budgets for our average fly.

\section{Thermal tolerance in starved and dehydrated flies}

To determine the amount of energy and water lost during measurements relative to the total budget of an individual fly, we first analysed how desiccation and starvation resistance changed as a function of temperature using data from Da Lage, Capy \& David (1989), who recorded survival times in desiccation and starvation resistance assays in D. melanogaster at nine temperatures ranging from $5{ }^{\circ} \mathrm{C}$ to $31^{\circ} \mathrm{C}$ (Fig. 3). If energy and water reserves are constant and ultimately determine the amount of time that an individual fly may cope with water or food deprivation, one would intuitively expect an inverse relationship between temperature and resistance to desiccation and starvation because of $\mathrm{Q}_{10}$ effects on metabolism. Comparisons between empirical values against predictions for our 'average' fly strongly support this prediction for starvation resistance and to a lesser extent, for desiccation resistance in the range of temperatures where deleterious effects of cold temperatures are not apparent (below a given threshold around $11-14^{\circ} \mathrm{C}$; Fig. 3). Estimates of desiccation 

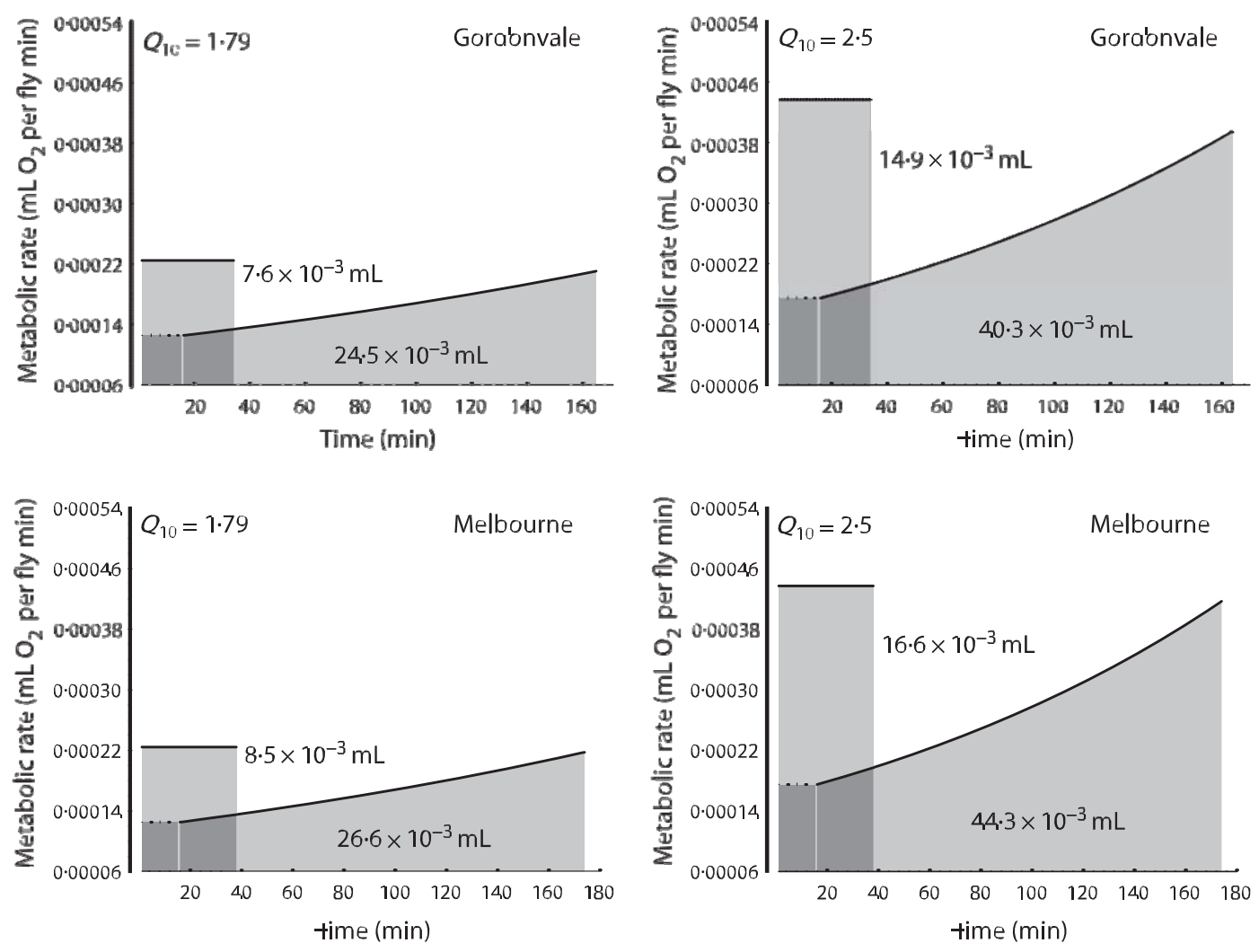

Fig. 2. Same as in Fig. 1 for the thermal resistance assays performed by Mitchell \& Hoffmann (2010) using two Australian populations of D. melanogaster (Gordonvale and Melbourne). The straight lines give the constant metabolic rates for the static assay at $38{ }^{\circ} \mathrm{C}$. The exponential lines are for the dynamic assays with $\mathrm{DT}=0\left\lfloor 06{ }^{\circ} \mathrm{C} \mathrm{min}^{3}\right.$. Shaded areas below the dotted points at the start of the ramping experiments are the estimated energy expenditures during the equilibration period of $15 \mathrm{~min}$ (also obviated for subsequent calculations in Table 1).
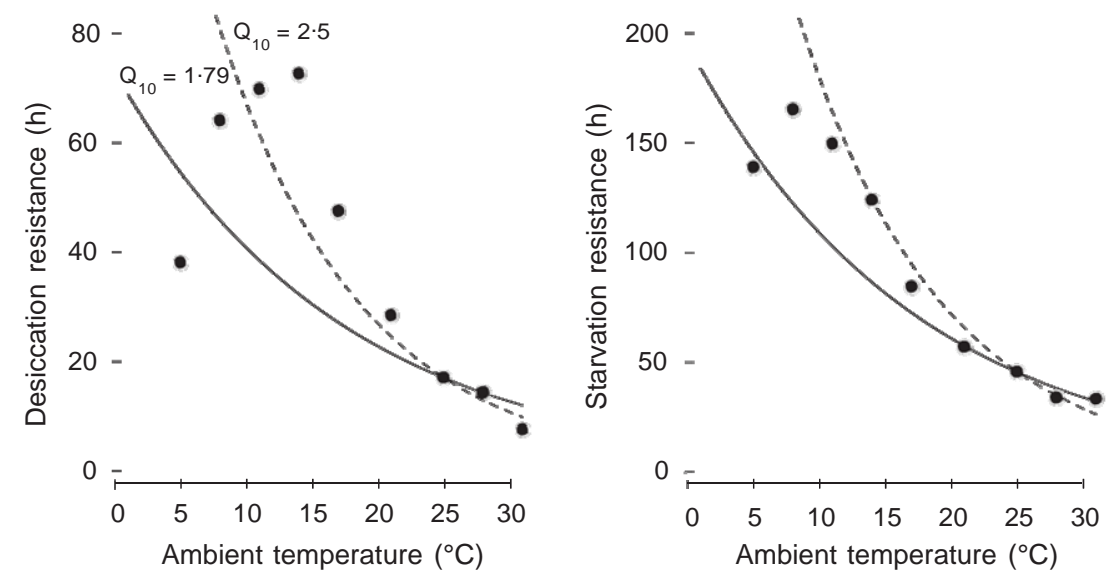

Fig. 3. Influence of temperature $\left(5,8,11,14,17,21,25,28\right.$ or $\left.31^{\circ} \mathrm{C}\right)$ on average survival times of D. melanogaster females in desiccation resistance and starvation resistance assays performed by Da Lage, Capy \& David (1989). Black dots are empirical values. Lines are the estimated energy expenditures extrapolated from an average fly weighting $1 \mathrm{mg}$ and metabolic rates of $\left.6 ! 31 \mathrm{~mL} \mathrm{O}_{2} \mathrm{~g}\right)^{1} \mathrm{~h}^{3}$ at $25^{\circ} \mathrm{C}$ with $\mathrm{Q}_{10}=1179$, or

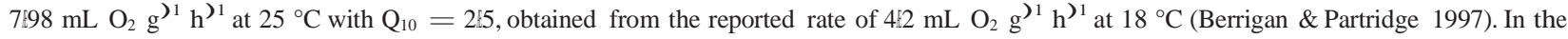
range of temperatures where deleterious effects of cold temperatures are not apparent (above $11-14{ }^{\circ} \mathrm{C}$ ) there is a reasonable fit with $\mathrm{Q}_{10}=2 ! 5$ for starvation resistance, but for desiccation resistance the fit improves assuming a higher $Q_{10}$.

resistance suggest that flies at lower temperatures are losing water at slower rates than predicted by metabolism (i.e. they tolerate desiccation for longer periods), which is likely due to the added effects of temperature on vapour pressure. Succinctly, the fraction of water that evaporates from respiratory organs and body surface should increase exponentially with temperature (e.g. at $38^{\circ} \mathrm{C}$ water vapour is about twice as high as at $25^{\circ} \mathrm{C}$; see Schmidt-Nielsen 1997, p. 324), resulting in a contribution to water loss that is independent of $\mathrm{Q}_{10}$ effects on metabolic rates. 
How much of an impact does the difference in energy expenditure calculated for different experimental protocols entail? At $25^{\circ} \mathrm{C}$, a D. melanogaster female can survive for approximately $4555 \mathrm{~h}$ in a water-saturated vial without food (starvation resistance), and $17 \mathrm{~h}$ when flies are water and food deprived (desiccation resistance; Da Lage, Capy \& David 1989). This would represent, respectively, a total energy expenditure of $287: 2-10^{3}$ or $107: 3-10^{3} \mathrm{~mL} \mathrm{O}_{2}$ during these trials with $\mathrm{Q}_{10}=1579$; and $362\left[9-10^{3}\right.$ or $1356 \cdot 10^{3}$ $\mathrm{mL} \mathrm{O}_{2}$ with $\mathrm{Q}_{10}=25$. As flies enclosed in sealed empty vials for the thermal tolerance assays somewhat match with the desiccation resistance protocol, it immediately follows that flies consumed about $10 \%$ of their energy reserves during measurements with slow heating rates, and between $20 \%$ and $30 \%$ of their water reserves by the time they are approaching $\mathrm{CT}_{\max }$ if we assume that respiratory rates of water loss are proportional to metabolism (Table 1).

These staggering results are actually conservative because they assume vapour pressure to remain constant across temperatures. If we repeat the previous analysis employing the $7: 5 \mathrm{~h}$ duration of the desiccation trial at $31^{\circ} \mathrm{C}$ (Da Lage, Capy \& David 1989), results would suggest that flies submitted to slow thermal ramping in Chown et al. (2009) and Mitchell \& Hoffmann (2010) might have lost around 40\% of their total water reserves (corresponding to $67: 1-10^{3}$ and $103: 7-10^{3} \mathrm{~mL} \mathrm{O}_{2}$ calculated assuming $\mathrm{Q}_{10}=1.79$ and $\mathrm{Q}_{10}=2: 5$ respectively) by the time they were knocked down. Interestingly, Levins (1969) showed that changes in heat tolerance in D. melanogaster following thermal acclimation could be partly explained by differences in water content: flies acclimated at higher temperatures would increase their mean body weight hence presumably water uptake.

Regardless of the exact amount of water lost, there is no doubt that flies are wasting a substantial fraction of their water reserves during long thermal tolerance assays (i.e. low DT), emphasizing that the effects of dehydration during ramping protocols to measure $\mathrm{CT}_{\max }$ should be taken into account. Analyses also suggest that physiological processes underlying energy and water economy become increasingly relevant in long assays of critical thermal limits, which is particularly true for $\mathrm{CT}_{\max }$ because temperatures increase in time. As we discuss below, these observations may have important repercussions for studies on thermal plasticity, and analysis of the genetic basis and evolution of thermal tolerance in natural populations.

\section{Plasticity, heritability and evolution of thermal tolerance limits}

No physiologist would find surprising that performance in general and thermal tolerance in particular, changes as a function of an individual's physical condition. This would explain, for instance, why flies in the static treatment of Mitchell \& Hoffmann (2010) were able to cope with $38^{\circ} \mathrm{C}$ for over 30 min while most individuals in the ramping treatment were knocked down at lower temperatures, resulting in an average $\mathrm{CT}_{\max }$ of $3619^{\circ} \mathrm{C}$ and $3715{ }^{\circ} \mathrm{C}$ for flies from Gordonvale and
Melbourne respectively. Flies in the ramping treatment were exhausted or dehydrated when approaching their 'basal target' $\mathrm{CT}_{\max }$, hence the general decrease in average $\mathrm{CT}_{\max }$ in longer trials (e.g. fig. 1 in Chown et al. 2009) probably reflects a real biological phenomenon with potentially important consequences in nature. However, effects of physical condition impinge on considerably more serious problems when attempting to obtain causal variance estimates of thermal tolerance limits or to study the effects of thermal acclimation on critical thermal limits. Subsequently, we illustrate these issues with a Gedankenexperiment.

\section{HERITABILITY/EVOLVABILITY}

Assume that we genetically engineer D. melanogaster isogenic flies to produce mutant alleles of target genes that affect only desiccation rates during the course of a heat resistance assay. Assume also that $\mathrm{CT}_{\max }$ under optimal physical conditions is $40{ }^{\circ} \mathrm{C}$ and that it decreases as individual flies become dehydrated at a linear rate of $005{ }^{\circ} \mathrm{C}$ per $1 \%$ of total water reserve lost. This is a reasonable scenario because $\mathrm{CT}_{\max }=$ 401) 0 047 - (1\% water lost) according to Chown et al's. (2009) experimental data and our estimated fraction of total metabolism spent in desiccation resistance assays with $\mathrm{Q}_{10}=215$ (Table 1). Incidentally, Huey et al. (1992, p. 492) noted that if heating rate is slow and if evaporative water loss is very high, then insects could be knocked down by desiccation, not by heat'; and Parsons (1980) had already shown that desiccated flies tolerate lower temperatures than their hydrated counterparts.

Notice that in our hypothetical scenario no variation exists on $\mathrm{CT}_{\max }$ per se apart from that imposed by dehydration, hence all individuals with the same water content should have identical $\mathrm{CT}_{\max }$. However, individuals will exhibit different $\mathrm{CT}_{\max }$ in heating assays simply because they lose water at different rates (Fig. 4). Consequently, one could erroneously conclude that the observed variation in $\mathrm{CT}_{\max }$ has a genetic basis when in reality it is due to variation in desiccation rates, which by definition is orthogonal to $\mathrm{CT}_{\max }$ because no genetic variation exists for upper thermal limits in our engineered flies (Falconer \& Mackay 1996). This might seem as a trivial point, but it is actually important because this environmental source of phenotypic variation in $\mathrm{CT}_{\max }$ scales up with experimental time (i.e. decreasing DT; Fig. 4).

Let us now turn the problem on its head, and ask what would happen if the phenotypic variance of $\mathrm{CT}_{\max }$ was solely due to (additive) genetic variation (i.e. assume that our Gedankenexperiment makes use of D. melanogaster isogenic flies targeted for genes that affect only $\mathrm{CT}_{\max }$ ). In this scenario, all individuals loose water at exactly the same rate and, therefore, observed variation in $\mathrm{CT}_{\max }$ at any given hydric condition reflects primarily genetic differences across individuals (e.g. individuals with a $\mathrm{CT}_{\max }$ corresponding to $41^{\circ} \mathrm{C}$ and $39^{\circ} \mathrm{C}$ in optimal conditions would exhibit a $\mathrm{CT}_{\max }$ of $40 \$ 5{ }^{\circ} \mathrm{C}$ and $3815{ }^{\circ} \mathrm{C}$, respectively, after losing $10 \%$ of their water reserves). Numerical results show that in this case the variance in $\mathrm{CT}_{\max }$ decreases with the duration of the assay, 

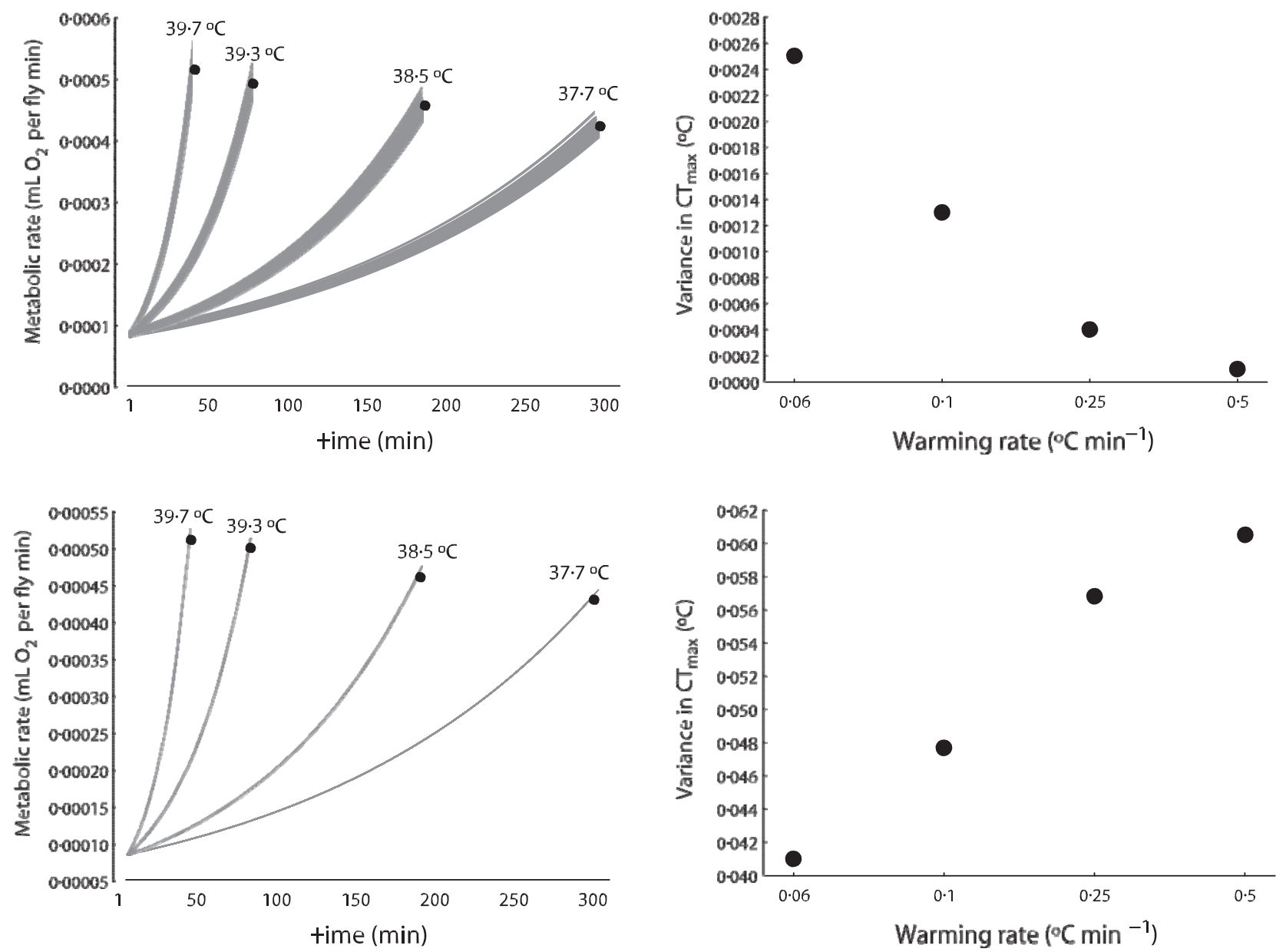

Fig. 4. $\mathrm{CT}_{\max }$ as a function of metabolic rates from numerical results assuming $100 \mathrm{D}$. melanogaster flies with: (a) (upper panels) fixed 'basal' $\mathrm{CT}_{\max }=40{ }^{\circ} \mathrm{C}$ and genetic variation for metabolic rates sampled from a normal distribution with (mean $\pm \mathrm{SD}$ ) $4 ! 2 \pm 0 ! 1 \mathrm{~mL} \mathrm{O}_{2} \mathrm{~g}^{\text {) }} \mathrm{h}^{3}{ }^{1}$ at $18{ }^{\circ} \mathrm{C}$; or (b) (lower panels) genetic variation for basal $\mathrm{CT}_{\max }$ also sampled from a normal distribution with parameters $40^{\circ} \mathrm{C} \pm 0 ! 25{ }^{\circ} \mathrm{C}$ and metabolic rates fixed at $4: 2 \mathrm{~mL} \mathrm{O} \mathrm{g}^{1}{ }^{1} \mathrm{~h}^{1}$ at $18^{\circ} \mathrm{C}^{\circ} \mathrm{CT}_{\max }$ is assumed to decrease as individuals flies become dehydrated at a linear rate of $0: 05{ }^{\circ} \mathrm{C}$ per $1 \%$ of total water reserve lost. Plotted are the metabolic rates in four ramping experiments with $\mathrm{T}_{0}=20^{\circ} \mathrm{C}$ and heating rates $\mathrm{DT}=0 \llbracket 06,0 \llbracket 1,0 \llbracket 25$ or $0 \leqq 5^{\circ} \mathrm{C} \mathrm{min}^{1}$, with time of the assay increasing with decreasing heating rate. Phenotypic variance in $\mathrm{CT}_{\max }$ is environmental (right upper panel) or genetic (right lower panel).

resulting in a positive association between heating rates and the genetic variance in $\mathrm{CT}_{\max }$ (Fig. 4). For example, in an assay with $\mathrm{T}_{0}=20{ }^{\circ} \mathrm{C}$ and $\mathrm{DT}=0.06{ }^{\circ} \mathrm{C}$ min ${ }^{1}$ a fly with a 'basal optimum' $\mathrm{CT}_{\max }=41{ }^{\circ} \mathrm{C}$ would be knocked down after $309 \mathrm{~min}$ at a recorded temperature of $38: 5{ }^{\circ} \mathrm{C}$, having lost $50: 2 \%$ of its water reserves. Another fly with an optimal $\mathrm{CT}_{\max }=39^{\circ} \mathrm{C}$, on the other hand, would be knocked down at $3659{ }^{\circ} \mathrm{C}$ after $282 \mathrm{~min}$, having lost $418 \%$ of its water content. Consequently, the genetically determined $2{ }^{\circ} \mathrm{C}$ difference in basal optimum $\mathrm{CT}_{\text {max }}$ drops to a $1: 6^{\circ} \mathrm{C}$ difference simply as a result of the slow heating rate, illustrating how the genetic variation in $\mathrm{CT}_{\max }$ changes with experimental conditions. We emphasize that this pattern reflects a decrease in (additive) genetic variance in the strict sense, and not an effect of genetic correlation $\mathrm{c}<1$ across $\mathrm{CT}_{\max }$ measured in different environmental conditions (see Falconer 1952; Falconer \& Mackay 1996, ch. 19). In fact, in our simulations the observed genetic correlation across environments remains consistently high (c 1).
As shown in Fig. 4, the thermal ramping rate affects the genetic and environmental components underlying the phenotypic variance of $\mathrm{CT}_{\max }$ in opposite directions. Considering that other factors such as thermal acclimation, variation in energy and water reserves, and measurement error also contribute to the phenotypic variation in $\mathrm{CT}_{\max }$, it is not surprising that similar protocols with different species (or vice versa) may result in contrasting outcomes. For instance, Chown et al. (2009) reported a decrease in phenotypic variances in $\mathrm{CT}_{\text {max }}$ with heating rates for the ant Linepithema humile, and the opposite trend for D. melanogaster (see their figs 2 and 3). In contrast, Mitchell \& Hoffmann (2010) showed that the phenotypic variation in knockdown time of D. melanogaster was three times higher in the ramping than in the static treatment. In the tsetse fly, Glossina pallidipes, tests of homoscedasticity indicate that the phenotypic variance in $\mathrm{CT}_{\max }$ was independent of the ramping protocol, but visual inspections suggest that the variance might be actually lower at higher heating rates (fig. 1a in Terblanche et al. 2007). Succinctly, 
no clear association between phenotypic variation and experimental protocol seems to emerge from empirical studies.

Nonetheless, the obvious implication of the previous numerical exercises is that both heritability $\left(\mathrm{h}^{2}=\mathbf{r}_{\mathrm{g}}^{2}\right.$

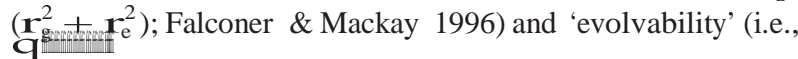
$\mathbf{r}_{\mathrm{g}}^{2}=\mathbf{I}^{2}$, where $\mathbf{l}$ is the average; Houle 1992) of $\mathrm{CT}_{\max }$ are expected to decrease at lower heating rates. Although the relative magnitudes of genetic $\left(\mathbf{r}_{\mathrm{g}}^{2}\right)$ and environmental $\left(\mathbf{r}_{\mathrm{e}}^{2}\right)$ variances will obviously depend on real values, we suspect $h^{2}$ should drop as heating rates decrease, primarily because of its disproportional impact on the environmental component. For instance, in our numerical results $\mathbf{r}_{\mathrm{e}}^{2}$ at a heating rate $\mathrm{D}=0106^{\circ} \mathrm{C} \mathrm{min}^{1}$ corresponds to $\mathbf{\mathbf { i } 2 7}$ times $\mathbf{r}_{\mathrm{e}}^{2}$ at

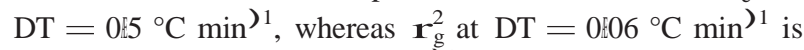
i 0 667 that at DT $\left.=0 \leqslant 5{ }^{\circ} \mathrm{C} \mathrm{min}\right)^{1}$ (Fig. 4). Employing these values and assuming that the contribution of $\mathbf{r}_{\mathrm{g}}^{2}$ and $\mathbf{r}_{\mathrm{e}}^{2}$ are equal at $\left.\mathrm{DT}=0: 5{ }^{\circ} \mathrm{C} \mathrm{min}\right)^{1}, \mathrm{~h}^{2}$ would drop to $0: 024$ at $\mathrm{DT}=0\left[06^{\circ} \mathrm{C} \mathrm{min}^{1}\right.$. It is also important to notice that the decrease in the adaptive potential of $\mathrm{CT}_{\max }$ with decreasing DT (Fig. 4) is also evident in evolvability estimates, which also drop by $\mathbf{i} 15 \%$ from the fastest to the slowest heating rates (this figure is quite consistent for different initial parameter values although it can slightly increase with a higher 'basal' $\mathrm{CT}_{\mathrm{MAX}}$ ). The scarce empirical available evidence supports this observation (Mitchell \& Hoffmann 2010): narrow sense $\mathrm{h}^{2}$ for knockdown times were relatively high and significant under static conditions (mean $\pm \mathrm{SE}: \mathrm{h}^{2}=0 \llbracket 22 \pm 0 \lll 07$ and $h^{2}=0: 14 \pm 0$ :05 for Gordonvale and Melbourne D. melanogaster populations, respectively), but were not significantly different from zero in ramping conditions (differences between protocols are listed in Table 1). Gilchrist \& Huey (1999) observation that knockdown temperatures in D. melanogaster is heritable and responds to selection partly supports our predictions, given that these authors employed a ramping protocol with fast heating rates $\left(\mathbf{\mathbf { 1 }} 1^{\circ} \mathrm{C} \mathrm{min}^{{ }^{1}}\right)$. It remains to be seen whether a similar experiment employing low heating rates would result in the same output.

Although we have assumed two extreme situations in the Gedankenexperiment (i.e. $\mathbf{r}_{\mathrm{g}}^{2}=0$ for $\mathrm{CT}_{\max }$ in the first scenarios and $\mathbf{r}_{\mathrm{e}}^{2}=0$ in the second) for illustrative purposes, there is ample evidence for the presence of genetic variation in both desiccation resistance and thermal limits in Drosophila populations (reviewed in Hoffmann \& Harshman 1999; Hoffmann, Sørensen \& Loeschcke 2003); albeit the seemingly lower capacity for genetic and plastic responses in $\mathrm{CT}_{\max }$ compared with $\mathrm{CT}_{\min }$ (Chown 2001; Hoffmann 2010). Moreover, the repeatability and heritability of standard metabolic rate is relatively high in several species of insects (Marais \& Chown 2003; Terblanche, Klok \& Chown 2004; Nespolo, Castaneda \& Roff 2007), including D. melanogaster (Williams, Rose \& Bradley 1997). In addition, desiccation and starvation resistance are genetically uncorrelated to metabolic rates in Drosophila (Djawdan, Rose \& Bradley 1997; Hoffmann \& Harshman 1999), hence our conclusion that the environmental component of the phenotypic variance for
$\mathrm{CT}_{\max }$ scales up with decreasing heating rates is probably applicable to real scenarios.

\section{PLASTICITY}

The preceding scenario also allows inquiring on the effect of heating rates on thermal acclimatory responses. Briefly, assume that, at temperatures above $25^{\circ} \mathrm{C}$ (the optimum for D. melanogaster), $\mathrm{CT}_{\max }$ increases in response to ramping conditions acclimation as

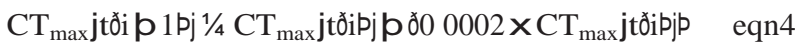

where $\mathrm{t}(\mathrm{i})$ is time at the ith minute after ambient temperature in the water-bath increased over $25^{\circ} \mathrm{C}$, and $\mathrm{CT}_{\max }|\mathrm{t}(0)|=40^{\circ} \mathrm{C}$ as above. We therefore make the implicit assumption that environment temperature (not heating rate) is the clue to trigger the thermal acclimatory responses.

Assuming $\mathrm{T}_{0}=20^{\circ} \mathrm{C}$ and that flies' physical condition is constant throughout the experiment (i.e. no desiccation occurs and, hence, $\mathrm{CT}_{\max }|\mathrm{t}(\mathrm{i})|$ remains equal to $\mathrm{CT}_{\text {max }}|\mathrm{t}(0)|$ when ambient temperature is $£ 25^{\circ} \mathrm{C}$ and thereafter increases as given by eqn 4 when $>25^{\circ} \mathrm{C}$ ), $\mathrm{CT}_{\max }$ at the end of the assays would increase from $40: 24^{\circ} \mathrm{C}$ with DT $=0: 5{ }^{\circ} \mathrm{C}$ $\min ^{1}$ to $42: 39{ }^{\circ} \mathrm{C}$ with DT $=006{ }^{\circ} \mathrm{C} \mathrm{min}^{3}$; that is, a nontrivial net beneficial effect of $2 \llbracket 15^{\circ} \mathrm{C}$ with the slowest heating rate. However, when desiccation effects are considered as in our first Gedankenexperiment (i.e. $\mathrm{CT}_{\max }|\mathrm{t}(\mathrm{i})|$ continuously decreases below $\mathrm{CT}_{\max }|\mathrm{t}(0)|$ because of desiccation effects when ambient temperature is $£ 25{ }^{\circ} \mathrm{C}$, and thereafter this decrease is somewhat compensated because of the thermal acclimatory responses given by eqn 4 when ambient temperature $>25^{\circ} \mathrm{C}$ ), the resulting $\mathrm{CT}_{\max }$ are $39: 88^{\circ} \mathrm{C}$ with $\mathrm{DT}=0: 5^{\circ} \mathrm{C} \min ^{1}$ and $39: 22$ with $\mathrm{DT}=0: 066^{\circ} \mathrm{C} \mathrm{min}^{1}$. What this numerical exercise shows is that desiccation can potentially overshadow thermal acclimation effects owing to short-term responses of individuals to changing temperatures (see also Woods \& Harrison 2002) and, more importantly, that a drop in $\mathrm{CT}_{\max }$ with slower heating rates does not necessarily rule out the 'beneficial acclimation hypothesis' (Leroi, Bennett \& Lenski 1994), which has some empirical support (Chung 1997; Nyamukondiwa \& Terblanche 2010; but see Huey et al. 1999).

Actually, in Drosophila pre-treatment or pre-exposure to a non-lethal high temperature enables survival at even higher temperatures (Levins 1969; Loeschcke, Krebs \& Barker 1994; Dahlgaard et al. 2002), and a similar response is documented for low temperatures (i.e. pre-exposure at non-lethal low temperatures enables survival at even lower temperatures; Kelty \& Lee 1999, 2001) thus suggesting that beneficial thermal acclimation does indeed happen. Parenthetically, when assessing $\mathrm{CT}_{\min }$ in ramping protocols and flies' metabolic costs were probably of little concern (Fig. 1), Chown et al. (2009) detected an important effect of beneficial acclimation: flies kept at $15^{\circ} \mathrm{C}$ had a $\mathrm{CT}_{\text {min }}$ 
that was $-215{ }^{\circ} \mathrm{C}$ below that of their corresponding counterparts raised at $25{ }^{\circ} \mathrm{C}$.

\section{Implications}

As discussed above, multiple variables can affect the outcome of ramping experiments aiming to estimate critical thermal limits. Regardless of the true nature of the variables (e.g. total energy expenditure, water loss, etc.), effects are likely cumulative and consequently increase with the duration of assays, everything else being equal (eqn 3). The obvious implication is that the longer the trial, the higher the contribution of these uncontrolled factors. Although we have focused on the consequences of ramping protocol on $\mathrm{CT}_{\max }$ given the conspicuous effects of increased temperatures on $\mathrm{Q}_{10}$, empirical observations suggest that energy reserves (triacylglycerols and proteins) are also important determinants of chilling tolerance in D. melanogaster (Chen \& Walker 1994; Misener, Chen \& Walker 2001). Empirical evidence and theoretical expectations suggest that flies' physical condition decays during the course of measurements, hence observed responses reflect the interaction of multiple stressors rather than the effects of temperature per se.

A related problem involves the interaction between genetic and acclimatory effects, because acclimatory responses (if any) should become increasingly important at slow heating rates. Our Gedankenexperiment suggests that the phenotypic expression of the 'basal' genetic diversity is expected to decrease under this experimental condition (Fig. 4), and the reduction might be even more pronounced if animals are acclimating during the course of the assay. The take-home message to circumvent all these problems is apparently straightforward: heating and cooling rates in ramping protocols should be as fast as possible (within the range in which measurement accuracy and individuals' physical condition are not affected) when the primary goal is to study the evolutionary potential of thermal tolerance or plastic responses to thermal acclimation. But this obviously raises another important question: what about ecological realism?

The fundamental issue regarding ecological realism is that heating or cooling rates in the laboratory may mimic conditions in nature (e.g. DT $\left.=0: 06{ }^{\circ} \mathrm{C} \mathrm{min}\right)^{1}$ roughly corresponds to the thermal increase experience by D. melanogaster flies during summer in arid environments of Australia; Mitchell \& Hoffmann 2010), but not necessarily the impact of other stressors. For instance, Drosophila adults can behaviourally thermoregulate (Dillon et al. 2009; Huey \& Pascual 2009; Rego et al. 2010) and might never encounter conditions similar to those of sealed empty vials in nature. Working in southeastern Spain, where ambient temperature in summer can be higher than $40{ }^{\circ} \mathrm{C}$ at midday, one of us (M. Santos, unpublished results) has observed that the heat tolerant cactophilic fly D. buzzatii remains mostly inside its feeding and breeding sites (rotting cladodes of Opuntia ficus-indica), and D. melanogaster can also be found inside rotting Opuntia fruits. These fruits are sweet and fleshy, and fermentation produces alcohol that can also be used as food by D. melanogaster (van
Herrewege \& David 1974). Although these flies could be experiencing a heat stress (Gibbs, Perkins \& Markow 2003; but see Feder, Roberts \& Bordelon 2000), food and water deprivation was probably not an issue as in the laboratory assays. It might even be the case that under these circumstances a low heating rate may result in higher $\mathrm{CT}_{\max }$, contrarily to what is observed in ramping protocols (Chown et al. 2009).

Conversely, there are situations when the thermal ramping protocols with slow heating rates do perfectly match field scenarios. For instance, tadpoles developing in temporary ponds experience natural heating rates around $0 \llbracket 03-0 £ 05{ }^{\circ} \mathrm{C} \mathrm{min}{ }^{1}$, and their $\mathrm{CT}_{\max }$ also drops at these values when compared with a higher but unrealistic heating rate of $150^{\circ} \mathrm{C}$ min ${ }^{1}$ (M. Tejedo, H. Duarte, M. Simon \& M. Katzenberger, unpublished data). Although food supply is kept constant in these assays, elevated temperatures increase metabolism and energetic demands in an exponential way because of $\mathrm{Q}_{10}$ effects, hence body conditions are likely to deteriorate over time (e.g. Mora \& Maya 2006) because of increased food demands and also because not all physiological processes scale up exponentially.

Nonetheless, given the potential contribution of other uncontrolled factors, even in these scenarios results stemming from measurements employing slow heating rates should be interpreted with caution. As our Gedankenexperiment shows, heritability may be severely underestimated because residual variation (regardless of its source) is expected to increase under these experimental conditions. Low heritability estimates would suggest a limited potential for critical thermal limits to evolve because the population will not track environmental changes, while in reality this result would partly reflect a lack of statistical power to detect the genetic source of variation underlying phenotypic variability in these traits (it is true, however, that the genetic variance is also expected to decrease; see Fig. 4). It is our contention here that the conclusion of a limited evolutionary potential for upper thermal limits when measured under 'realistic ecological' settings needs to be revised, and its implications more critically addressed. We find ourselves in the uncomfortable situation where the common observation that heat tolerance varies geographically in an 'adaptive' direction (Levins 1969; Coyne, Bundgaard \& Prout 1983; Hoffmann, Anderson \& Hallas 2002; Anderson et al. 2003; Angilletta et al. 2007) is at odds with this conclusion.

\section{Concluding remarks}

As our results highlight, physiological traits are inherently variable and susceptible to be affected by experimental conditions, and the establishment of a formal conceptual framework may help designing better experiments and also elucidating which factors might explain observed empirical patterns (see also Angilletta 2009). With the application of a simple theoretical model, we have illustrated how the trivial premise that measurement duration should eventually have an impact on organisms' physical condition can affect the experimental outcome of dynamic methods to study critical thermal limits. In this context, it is important to 
emphasize that our recommendation to employ relatively rapid ramping conditions is particularly relevant for studies on the genetic basis of phenotypic variation and/or the effects of thermal acclimation on critical thermal limits. For certain study models, this observation unavoidably results in a trade-off between methodological adequacy vs. ecological realism (a physiological analogue of Heisenberg's uncertainty principle). Fast ramping rates may not be ecologically realistic in some instances, but they minimize the contribution of uncontrolled variables in experimental settings. Conversely, slow ramping rates may closely mimic conditions in nature, but the effects of other factors become increasingly important and may ultimately overshadow the effects of the target variables under study.

The fundamental message we want to stress is that different ramping protocols ultimately result in variation across a multitude of factors, hence researchers should be extremely cautious when interpreting results obtained under different experimental conditions and analysing their potential biological significance. For instance, when discussing their findings with the tsetse fly G. pallipides, Terblanche et al. (2007, p. 2939) observed that $T_{0}$ and DT affect and interact with thermal limits, and tried to test their effects, as well as the mean duration of the assay, by entering all variables sequentially in a linear model. The problem here is that these three variables are necessarily co-linear due to the relationship $\mathrm{t} 1 / 4 \mathrm{dCT}_{\max }-\mathrm{T}_{0} \mathrm{p}=\mathrm{DT}$. It is well known that (severe) co-linearity creates serious problems if the purpose of the regression is to understand the process, to identify important variables in the process, or to obtain meaningful estimates of the regression coefficients (Rawlings 1988; Hadi \& Ling 1998; Jolliffe 2004). Although any of those variables could be taken as a surrogate for the whole set (Thisted 1980), a simple scaling up of metabolic costs with decreasing heating rates may explain why differences in $\mathrm{CT}_{\max }$ are associated with exposure duration. Note that we are not saying that energy expenditure is the only factor that matters; instead, we simply stress that effects such as the nearly fourfold difference in energy consumption between measurements in D. melanogaster (Table 1) are probably not negligible and may be widespread across study systems.

\section{Acknowledgements}

We thank the associate editor and two anonymous reviewers for providing many constructive and thought-provoking comments on an earlier draft that helped to significantly improve the manuscript. E.L.R. is supported by a Ramon y Cajal contract and by grant BFU2009-07564 from the Ministerio de Ciencia e Innovacion (Spain). M.T. is supported by grant CGL2009-12767C02-02 from the Ministerio de Ciencia e Innovacion. M.S. is supported by grants CGL2009-12912-C03-01 and CGL2010-15395 from the Ministerio de Ciencia e Innovacion. Financial support by grant 2009SGR 636 from Generalitat de Catalunya to the Grup de Biologia Evolutiva is also gratefully acknowledged.

\section{References}

Anderson, A.R., Collinge, J.E., Hoffmann, A.A., Kellet, M. \& McKechnie, S.W. (2003) Thermal tolerance trade-offs associated with the right arm of chromosome 3 and marked by hsr-omega gene in Drosophila melanogaster. Heredity, 90, 195-202.

Angilletta, M.J. (2009) Thermal Adaptation. A Theoretical and Empirical Synthesis. Oxford University Press, Oxford.

Angilletta, M.J., Wilson, R.S., Niehaus, A.C., Sears, M.W., Navas, C.A. \& Ribeiro, P.L. (2007) Urban physiology: city ants possess high heat tolerance. PLoS One, 2, e258.

Berrigan, D. \& Partridge, L. (1997) Influence of temperature and activity on the metabolic rate of adult Drosophila melanogaster. Comparative Biochemistry and Physiology, 118A, 1301-1307.

Chen, C.P. \& Walker, V.K. (1994) Cold-shock and chilling tolerance in Drosophila. Journal of Insect Physiology, 40, 661-669.

Chown, S.L. (2001) Physiological variation in insects: hierarchical levels and implications. Journal of Insect Physiology, 47, 649-660.

Chown, S.L., Jumbam, K.R., Sørensen, J.G. \& Terblanche, J.S. (2009) Phenotypic variance, plasticity and heritability estimates of critical thermal limits depend on methodological context. Functional Ecology, 23, 133-140.

Chung, K.S. (1997) Tolerancia termica del pez tropical Cyprinodon dearborni (Atheriniformes: Cyprinodontidae) a diversas tasas de calentamiento y salinidades. Revista de Biología Tropical, 45, 1541-1545.

Clark, P.L. (2004) Protein folding in the cell: reshaping the folding funnel. Trends in Biochemical Sciences, 29, 527-534.

Cowles, R. \& Bogert, C.M. (1944) A preliminary study of the thermal requirements of desert reptiles. Bulletin of the American Museum of Natural History, 83, 261-296.

Coyne, J.A., Bundgaard, J. \& Prout, T. (1983) Geographic variation of tolerance to environmental stress in Drosophila pseudoobscura. American Naturalist, 122, 474-488.

Da Lage, J-L., Capy, P. \& David, J-R. (1989) Starvation and desiccation tolerance in Drosophila melanogaster adults: effects of environmental temperature. Journal of Insect Physiology, 35, 453-457.

Dahlgaard, J., Loeschcke, V., Michalak, P. \& Justensen, J. (2002) Induced thermotolerance and associated expression of the heat-shock protein Hsp70 in adult Drosophila melanogaster. Functional Ecology, 12, 786-793.

Deutsch, C.A., Tewksbury, J.J., Huey, R.B., Sheldon, K.S., Ghalambor, C.K., Haak, D.C. \& Martin, P.R. (2008) Impacts of climate warming on terrestrial ectotherms across latitude. Proceedings of the National Academy of Sciences of the USA, 105, 6668-6672.

Dillon, M.E., Wang, G., Garrity, P.A. \& Huey, R.B. (2009) Thermal preference in Drosophila. Journal of Thermal Biology, 34, 109-119.

Djawdan, M., Rose, M.R. \& Bradley, T.J. (1997) Does selection for stress resistance lower metabolic rate? Ecology, 78, 828-837.

Falconer, D.S. (1952) The problem of environment and selection. American Naturalist, 86, 293-298.

Falconer, D.S. \& Mackay, T.F.C. (1996) Introduction to Quantitative Genetics. Longman Group Limited, Harlow.

Feder, M.E., Roberts, S.P. \& Bordelon, A.C. (2000) Molecular thermal telemetry of free-ranging adult Drosophila melanogaster. Oecologia, 123, 460-465.

Gibbs, A.G., Perkins, M.C. \& Markow, T.A. (2003) No place to hide: microclimates of desert Drosophila. Journal of Thermal Biology, 28, 353-362.

Gilchrist, G.W. \& Huey, R.B. (1999) The direct response of Drosophila melanogaster to selection on knockdown temperature. Heredity, 83, 15-29.

Gillooly, J.F., Brown, J.H., West, G.B., Savage, V.M. \& Charnov, E.L. (2001) Effects of size and temperature on metabolic rate. Science, 293, 2248-2251.

Hadi, A.S. \& Ling, R.F. (1998) Some cautionary notes on the use of principal components regression. American Statistician, 52, 15-19.

Haldane, J.B.S. (1940) Keeping Cool and Other Essays. Chatto \& Windus, London.

van Herrewege, J. \& David, J. (1974) Utilisation de l'alcohol ethylique dans le metabolisme energetique d'un insecte: influence sur la duree de survie des adultes de Drosophila melanogaster. Comtes Rendus de Academie des Sciences, Serie D, 279, 335-338.

Hochachka, P.W. \& Somero, G.N. (2002) Biochemical Adaptation. Mechanism and Process in Physiological Evolution. Oxford University Press, New York.

Hoffmann, A.A. (2010) Physiological climatic limits in Drosophila: patterns and implications. Journal of Experimental Biology, 213, 870-880.

Hoffmann, A.A., Anderson, A. \& Hallas, R. (2002) Opposing clines for high and low temperature resistance in Drosophila melanogaster. Ecology Letters, 5, 614-618.

Hoffmann, A.A. \& Harshman, L.G. (1999) Desiccation and starvation resistance in Drosophila: patterns of variation at the species, population and intrapopulation levels. Heredity, 83, 637-643.

Hoffmann, A.A., Sørensen, J.G. \& Loeschcke, V. (2003) Adaptation of Drosophila to temperature extremes: bringing together quantitative and molecular approaches. Journal of Thermal Biology, 28, 175-216. 
Houle, D. (1992) Comparing the evolvability and variability of quantitative traits. Genetics, 130, 195-204.

Huey, R.B. \& Kingsolver, J.G. (1989) Evolution of thermal sensitivity of ectotherm performance. Trends in Ecology and Evolution, 4, 131-135.

Huey, R.B. \& Pascual, M. (2009) Partial thermoregulatory compensation by a rapidly evolving invasive species along a latitudinal cline. Ecology, 90, 17151720 .

Huey, R.B. \& Stevenson, R.D. (1979) Integrating thermal physiology and ecology of ectotherms: discussion of approaches. American Zoologist, 19, 357.

Huey, R.B., Crill, W.D., Kingsolver, J.G. \& Weber, K.E. (1992) A method for rapid measurement of heat or cold resistance of small insects. Functional Ecology, 6, 489-494.

Huey, R.B., Berrigan, D., Gilchrist, G.W. \& Herron, J.C. (1999) Testing the adaptive significance of acclimation: a strong inference approach. American Zoologist, 39, 323-336.

Huey, R.B., Deutsch, C.A., Tewksbury, J.J., Vitt, L.J., Hertz, P.E., Alvarez Perez, H.J. \& Garland Jr, T. (2009) Why tropical forest lizards are vulnerable to climate warming. Proceedings of the Royal Society B: Biological Sciences, 276, 1939-1948.

Jolliffe, I.T. (2004) Principal Component Analysis, 2nd edition. Springer, New York.

Kelty, J.D. \& Lee, R.E. (1999) Induction of rapid cold hardening by cooling at ecologically relevant rates in Drosophila melanogaster. Journal of Insect Physiology, 45, 719-726.

Kelty, J.D. \& Lee, R.E. (2001) Rapid cold-hardening of Drosophila melanogaster (Diptera: Drosophilidae) during ecologically based thermoperiodic cycles. Journal of Experimental Biology, 204, 1659-1666.

Leroi, A.M., Bennett, A.E. \& Lenski, R.E. (1994) Temperature acclimation and competitive fitness: an experimental test of the beneficial acclimation assumption. Proceedings of the National Academy of Sciences of the USA, 91, 1917-1921.

Levins, R. (1969) Thermal acclimation and heat resistance in Drosophila species. American Naturalist, 103, 483-499.

Loeschcke, V., Krebs, R.A. \& Barker, J.S.F. (1994) Genetic variation for resistance and acclimation to high temperature stress in Drosophila buzzatii. Journal of the Linnean Society, 52, 83-92.

Marais, E. \& Chown, S.L. (2003) Repeatability of standard metabolic rate and gas exchange characteristics in a highly variable cockroach, Perisphaeria sp. Journal of Experimental Biology, 206, 4565-4574.

Martin, T.L. \& Huey, R.B (2008) Why "suboptimal” is optimal: Jensen's inequality and ectotherm thermal preferences. American Naturalist, 171, E102-E118.

Misener, S.R., Chen, C.P. \& Walker, V.K. (2001) Cold tolerance and proline metabolic gene expression in Drosophila melanogaster. Journal of Insect Physiology, 47, 393-400.

Mitchell, K.A. \& Hoffmann, A.A. (2010) Thermal ramping rate influences evolutionary potential and species differences for upper thermal limits in Drosophila. Functional Ecology, 24, 694-700.

Mora, C. \& Maya, M.F. (2006) Effect of the rate of temperature increase of the dynamic method on the heat tolerance of fishes. Journal of Thermal Biology, 31, 337-341

Nespolo, R.F., Castaneda, L.E. \& Roff, D.A. (2007) Quantitative genetic variation of metabolism in the nymphs of the sand cricket, Gryllus firmus, inferred from an analysis of inbred lines. Biological Research, 40, 5-12.

Nyamukondiwa, C. \& Terblanche, J.S. (2010) Within-generation variation of critical thermal limits in adult Mediterranean and Natal fruit flies Ceratitis capitata and Ceratitis rosa: thermal history affects short-term responses to temperature. Physiological Entomology, 35, 255-264.

Parsons, P.A. (1980) Parallel climatic races for tolerances to high temperaturedesiccation stress in two Drosophila species. Journal of Biogeography, 7, 97101

Peck, L.S., Clark, M.S., Morley, S.A., Massey, A. \& Rossetti, H. (2009) Animal temperature limits and ecological relevance: effects of size, activity and rates of change. Functional Ecology, 23, 248-256.

Rawlings, J.O. (1988) Applied Regression Analysis. Wadsworth \& Brooks / Cole, Pacific Grove.

Rego, C., Balanya, J., Fragata, I., Matos, M., Rezende, E.L. \& Santos, M. (2010) Clinal patterns of chromosomal inversion polymorphisms in Drosophila subobscura are partly associated with thermal preferences and heat stress resistance. Evolution, 64, 385-397.

Schmidt-Nielsen, K. (1997) Animal Physiology. Adaptation and Environment, 5th edition. Cambridge University Press, Cambridge.

Sinervo, B., Mendez-de-la-Cruz, F., Miles, D.B., Heulin, B., Bastiaans, E., Villagran-Santa Cruz, M. et al. (2010) Erosion of lizard diversity by climate change and altered thermal niches. Science, 328, 894-899.

Terblanche, J.S., Klok, C.J. \& Chown, S.L. (2004) Metabolic rate variation in Glossina pallidipes (Diptera: Glossinidae): gender, ageing and repeatability. Journal of Insect Physiology, 50, 419-428.

Terblanche, J.S., Deere, J.A., Clusella-Trullas, S., Janion, C. \& Chown, S.L. (2007) Critical thermal limits depend on methodological context. Proceedings of the Royal Society B, 274, 2935-2942.

Tewksbury, J.J., Huey, R.B. \& Deutsch, C.A. (2008) Putting the heat on tropical animals. Science, 320, 1296-1297.

Thisted, R.A. (1980) Comment: a critique of some ridge regression methods. Journal of the American Statistical Association, 75, 81-86.

Williams, A.E., Rose, M.R. \& Bradley, T.J. (1997) $\mathrm{CO}_{2}$ release patterns in Drosophila melanogaster: the effect of selection for desiccation resistance. Journal of Experimental Biology, 200, 615-624

Woods, H.A. \& Harrison, J.F. (2002) Interpreting rejections of the beneficial acclimation hypothesis: when is physiological plasticity adaptive? Evolution, 56, 1863-1866.

Received 1 June 2010; accepted 16 August 2010

Handling Editor: Michael Angilletta

\section{Supporting Information}

Additional supporting information may be found in the online version of this article.

Appendix S1. Thermal effects on metabolism employing other $\mathrm{Q}_{10}$ functions.

Fig. S1. $\mathrm{Q}_{10}$ as a function of temperature using eqn S1.

As a service to our authors and readers, this journal provides supporting information supplied by the authors. Such materials may be re-organized for online delivery, but are not copy-edited or typeset. Technical support issues arising from supporting information (other than missing files) should be addressed to the authors. 